\title{
Influence of a Single Ether Bond on Assembly, Orientation, and Miscibility of Phosphocholine Lipids at the Air-Water Interface
}

Received 00th January 20xx, Accepted 00th January 20xx DOI: $10.1039 / \times 0 \times x 00000 x$

\author{
Matthias Hoffmann, ${ }^{\mathrm{a}, \mathrm{b}}$ Simon Drescher, ${ }^{\mathrm{c}, \mathrm{d}}$ Christian Schwieger ${ }^{* \mathrm{~b}}$ and Dariush Hinderberger *a,b
}

How does a small change in the structure of a phospholipid affect its supramolecular assembly? In aqueous suspensions, the substitution of one ester linkage in DPPC (1,2-dipalmitoyl-sn-glycero-3-phosphocholine) by an ether linkage alters its phase behaviour completely. To unravel the effect of replacing a phospholipid's ester linkage by an ether linkage in lipid monolayers, we characterized pure monolayers of the model lipid DPPC and its sn-2 ether analogue PHPC (1-palmitoyl-2-Ohexadecyl-sn-glycero-3-phosphocholine) as well as mixtures of both by measurements of surface pressure - molecular area $\left(\pi-A_{\mathrm{mol}}\right)$ isotherms. In addition, we used infrared reflection absorption spectroscopy (IRRAS) to study lipid condensation, lipid chain orientation, headgroup hydration, and lipid miscibility in all samples. Mixed monolayers consisting of DPPC and PHPC were studied further using epifluorescence microscopy. Our results indicate a strong influence of the $s n-2$ ether linkage on headgroup hydration and ordering effects in the regions of the apolar chains and the headgroups. Both effects could originate from changes in glycerol conformation. Furthermore, we observed a second plateau in the $\pi-A_{\mathrm{mol}}$ isotherms of DPPC/PHPC mixtures and analysis of the mixed $\pi-A_{\text {mol }}$ isotherms reveals a non-ideal mixing behaviour of both lipids which may be caused by conformational differences in their headgroups.

\section{Introduction}

In nature, ether lipids are highly abundant in Archaea. ${ }^{1}$ Many representatives of this domain of life are extremophiles, needing extraordinarily stable membranes to withstand their extreme habitat conditions, e.g. at extremely low $\mathrm{pH}$, high temperature, or high ionic strength. ${ }^{1}$ To maintain a functional membrane structure, ester lipids are not suitable. In this environment, chemically more stable ether lipids as well as membrane-spanning bipolar tetraether lipids (TELs), also known as bolaamphiphiles, ${ }^{2}$ have evolved. ${ }^{3,4}$

In biophysics of lipids, it is well known that ether and ester lipids exhibit different phase behaviours. In aqueous suspensions of some ether lipids, a complex thermotropic polymorphism is observed. ${ }^{5}$ When comparing 1,2-dipalmitoyl-snglycero-3-phosphocholine (DPPC) with its mono- or di-ether derivatives, namely 1-palmitoyl-2-O-hexadecyl-sn-glycero-3phosphocholine (PHPC) and 1,2-di-O-hexadecyl-sn-glycero-3phosphocholine (DHPC), respectively, significant differences in gel phase structures are found (see the Supporting Information for the molecular structures). While DPPC forms crystalline $\left(L_{c}\right)$,

a. Institute of Chemistry, Martin Luther University (MLU) Halle-Wittenberg, VonDanckelmann-Platz 4, 06120 Halle (Saale), Germany.

b. Interdisciplinary Research Center HALOmem, MLU Halle-Wittenberg, Charles Tanford Protein Center, Kurt-Mothes-Str. 3a, 06120 Halle (Saale), Germany. Institute of Pharmacy, MLU Halle-Wittenberg, Wolfgang-Langenbeck-Str. 4, 06120 Halle (Saale), Germany.

d. Phospholipid Research Center, Im Neuenheimer Feld 515, 69120 Heidelberg, Germany.

Electronic Supplementary Information (ESI) available: Synthetic details, furthe IRRAS data, simulation of IRRA spectra, further PCA results and thermodynamic data. lamellar gel $\left(L_{\beta}{ }^{\prime}\right)$, and rippled gel $\left(P_{\beta}{ }^{\prime}\right)$ phases at temperatures below the main transition temperature $\left(T_{\mathrm{m}}\right)$ of $41.6{ }^{\circ} \mathrm{C}$, PHPC and DHPC form a lamellar interdigitated gel phase $L_{\beta}$ l (and DHPC an additional $L_{\beta}$ phase between 34.8 and $43.9{ }^{\circ} \mathrm{C}$ ) at temperatures below $T_{\mathrm{m}}{ }^{5}$ In PHPC and DHPC, one or two small changes in the region linking the alkyl chain and headgroup, i.e. the glycerol backbone, cause major structural modifications of their supramolecular assemblies in comparison with DPPC. Lewis et al. studied the reasons for the differences between these otherwise structurally identical lipids extensively using infrared (IR) spectroscopy and isotopic labeling. ${ }^{5}$ They concluded that the exchange of at least one ester bond with an ether bond induces a conformational change of the involved glycerol backbone, altered hydration of the remaining carbonyl moiety, and conformational changes of the adjacent chain segment. ${ }^{5}$ Hence, a change in glycerol orientation caused by as little as the substitution of one ester bond with an ether bond enables the lipid to preferably aggregate in a lipid gel phase showing alkyl chain interdigitation at temperatures below $T_{\mathrm{m}}$. Consequently, in aqueous suspensions the effects of ether bonds on lipid properties are understood quite well.

In contrast, differences between DPPC and PHPC in Langmuir monolayers are far less studied and understood. Surface pressure - molecular area $\left(\pi-A_{\mathrm{mol}}\right)$ isotherms of DPPC, PHPC, and DHPC were already measured and characterized by fluorescence microscopy and X-ray diffraction. ${ }^{6-8}$ Brezesinski et al. observed a decreased chain tilt angle of DHPC and PHPC, in comparison to DPPC. Moreover, the lateral lipid density of both ether lipids is increased compared to DPPC monolayers. ${ }^{6}$ They predicted a change in glycerol conformation to be responsible for these differences and the hydration of the headgroup to 
change. ${ }^{6}$ However, this remains speculation until now and little is known about structure, conformation, and hydration of the glycerol backbone of ether lipids in monolayers.

Here, we first investigate the phase behaviour, lipid conformation, and hydration of DPPC and PHPC monolayers, respectively. We present $\pi-A_{\text {mol }}$ isotherms combined with infrared reflection absorption (IRRA) spectra to detect how monolayers of both lipids differ in their molecular changes caused by compression of the monolayer. We evaluate the chain order parameter $\left.\left(\mathrm{S}_{(\mathrm{C}}\right)\right)$ and the order parameter of the phosphate group $\left.\left(\mathrm{S}_{\left(\mathrm{PO}_{2}^{-}\right.}\right)\right)$by performing a least square minimization of both methylene stretching vibrational bands and the antisymmetric phosphate stretching vibrational band, respectively. To characterize the hydration of the carbonyl moiety of both lipids, we use principal component analysis (PCA).

In the second part of this study, we report the characterization of mixed monolayers of DPPC and PHPC, in particular their miscibility at the air-water interface, by epifluorescence microscopy using a rhodamine-labelled lipid, the surface phase rule, and calculation of the excess Gibbs energy of mixing $\left(\Delta G_{\text {exc }}\right)$ and the Gibbs energy of mixing $\left(\Delta G_{\text {mix }}\right) .{ }^{9,} 10$ In addition, we present IRRA spectra of mixtures of PHPC and DPPC- $d_{62}$, bearing fully deuterated alkyl chains, to characterize their miscibility spectroscopically.

\section{Experimental}

\section{Materials}

1,2-Dipalmitoyl-sn-glycero-3-phosphocholine (DPPC) was purchased from Genzyme Pharmaceuticals (Cambridge, MA, USA) and used without further purification. 1-Palmitoyl-2-Ohexadecyl-sn-glycero-3-phosphocholine (PHPC) was synthesized as described in the Supporting Information. DPPC bearing perdeuterated acyl chains (DPPC- $d_{62}$ ) was purchased from Avanti Polar Lipids Inc. (Alabaster, AL, USA). The fluorescent dye 1,2-dipalmitoyl-sn-glycero-3-phosphoethanolamine- $\mathrm{N}$-(lissamine rhodamine $B$ sulfonyl) (ammonium salt) (Rh-DPPE) was obtained from Life Technologies $\mathrm{GmbH}$ (Darmstadt, Germany). The solvents chloroform and methanol (HPLC-grade) were obtained from Carl Roth (Karlsruhe, Germany).

\section{Methods}

Sample preparation. All used lipids were dissolved in chloroform/ methanol $(9 / 1, v / v)$. Mixtures of phospholipids were prepared by mixing different amounts of appropriate lipid stock solutions using glass syringes (Hamilton Bonaduz, Bonaduz, Switzerland). For storage, solvents were then removed in a gentle stream of nitrogen. Directly prior to experiments, the lipids and lipid mixtures were dissolved again in chloroform/methanol.

Monolayer preparation. All $\pi-A_{\mathrm{mol}}$ isotherms were measured on a rectangular Teflon trough $\left(78.3 \times 6.8 \mathrm{~cm}^{2}\right.$ Riegler \& Kirstein, Potsdam, Germany) except for compression isotherm measurements with parallel IRRAS detection. The Langmuir trough was equipped with a Wilhelmy sensor and filled with $\mathrm{H}_{2} \mathrm{O}$ (MilliQ Millipore water with a specific resistance of $\rho=18.2 \mathrm{M} \Omega \mathrm{cm}$ ). After the trough was filled with water, the freshly dissolved lipids or lipid mixtures were carefully spread on the surface with a glass syringe (Hamilton Bonaduz, Bonaduz, Switzerland). The solvents were allowed to evaporate for at least 10 min prior to each measurement.

Monolayer compression. All shown $\pi-A_{\text {mol }}$ isotherms were compressed at the air-water interface by Teflon barriers moving at a compression speed of $2 \AA^{2}$ (molecule min)-1. During the measurements, 40 points per seconds were averaged. In all measurements, the temperature of subphase and monolayer was kept constant (accuracy $\Delta T \pm 0.2 \mathrm{~K}$ ) through a coupled water-cooling system operated at $20^{\circ} \mathrm{C}$. Each isotherm was measured at least three times, from which one representative isotherm is shown.

Analysis of Langmuir isotherms. From each measured isotherm, the monolayer compressibility $C_{\mathrm{S}}$ was evaluated:

$$
C_{S}=-\frac{1}{A_{m o l}}\left(\frac{d A_{m o l}}{d \pi}\right)_{T}
$$

to determine the transition surface pressure $\pi_{\text {plateau }}$ from the maximal value of $C_{s}$.

The mixing behaviour of the lipids was studied as follows. Thermodynamically, the Gibbs energy of mixing $\Delta G_{\text {mix }}$ describes whether the components of a mixture are miscible or not. It can be split in an ideal and an excess, i.e. non-ideal, term:

$$
\Delta G_{\text {mix }}=\Delta G_{i d}+\Delta G_{\text {exc }}
$$

$\Delta G_{\text {id }}$ only depends on entropy, specifically on mixture composition, that is the mole fraction $x_{i}$, and temperature. This value is always negative, which means that, ideally, all components are miscible regardless of composition:

$$
\Delta G_{i d}=R T \sum_{i} x_{i} \ln \left(x_{i}\right)
$$

However, demixing can occur. Deviations from ideal behaviour are included in $\Delta G_{\text {exc. }}$ In two-dimensional systems such as Langmuir monolayers, deviations from ideal mixing are associated with an excess areas $A_{\text {exc. }}$.

$$
\Delta G_{\text {exc }}=\int_{0}^{\pi} A_{\text {exc }}(\pi) d \pi
$$

with

$$
A_{\text {exc }}(\pi)=A_{\text {mol,measured }}(\pi)-\sum_{i} x_{i} A_{\text {mol, } i}(\pi)
$$

The integration of (4) was conducted from $\pi=0 \mathrm{mN} \mathrm{m}^{-1}$, starting in the gaseous/liquid-expanded (LE) transition region. Combination of $\Delta G_{\text {id }}$ and $\Delta G_{\text {exc }}$ results in:

$$
\Delta G_{m i x}=R T \sum_{i} x_{i} \ln \left(x_{i}\right)+\int_{0}^{\pi} A_{\text {exc }}(\pi) d \pi
$$

Infrared reflection absorption spectroscopy (IRRAS). According to a procedure described elsewhere, ${ }^{11}, 12$ IRRAS experiments were performed on a Bruker Vector 70 FT-IR spectrometer equipped with an $\mathrm{A} 511$ reflection unit (Bruker Optics, Ettlingen, 
Germany), a liquid nitrogen-cooled MCT detector, and a Langmuir trough system (Riegler \& Kirstein, Potsdam, Germany). The trough system consists of a rectangular sample trough $\left(30 \times 6 \mathrm{~cm}^{2}\right)$ and a small circular reference trough $(r=3 \mathrm{~cm})$. Surface pressure in the sample trough was detected with a Wilhelmy sensor using a filter paper as the pressure probe. During the measurements, the filling levels of both troughs were kept equal and constant by means of an automated, laser-reflection-controlled pumping system connected to a reservoir of $\mathrm{H}_{2} \mathrm{O}$. Prior to collection of each monolayer IRRA spectrum, a spectrum of the pure subphase was measured with identical conditions to ensure best comparability and effective water vapor compensation.

Two general types of experiments were performed in this work: (i) IRRAS measurements at constant angle of incidence and polarization during compression of the monolayer and (ii) angle- and polarization-dependent IRRAS measurements to reveal orientations of different molecular moieties. For (i), IRRA spectra were collected at a constant angle of incidence $\varphi=60^{\circ}$ and with s-polarized IR light. 1000 single interferograms were collected, averaged, and, subsequently, Fourier-transformed with a zero-filling factor of 2 to obtain one spectrum with a nominal spectral resolution of $2 \mathrm{~cm}^{-1}$. Here, five of these individual spectra were averaged to obtain one final spectrum to ensure excellent signal to noise ratio. For (ii), we varied both, angle of incidence (25 to $70^{\circ}$ in increments of $3^{\circ}$ ) and polarization (s- and p-polarization) of the incoming IR beam. Either 1000 (s-polarized IR light) or 2000 (p-polarized IR light) interferograms were averaged and Fourier-transformed with the same parameters as described above. At least three of the resulting spectra were averaged to acquire the shown spectra. The $\pi-A_{\mathrm{mol}}$ isotherms of DPPC and PHPC, respectively, were halted at four different surface pressures at 3,10, 20, and $30 \mathrm{mN} \mathrm{m}^{-1}$ ) and sets of polarization- and angle-dependent IRRA spectra were measured at each pressure, which allows comparison of different compression states of both lipids.

Simulation and fitting of the experimental angle- and polarization-dependent IRRA spectra was conducted as explained in detail elsewhere. ${ }^{13-15}$

Principal component analysis (PCA). The goal of a principal component analysis (PCA) is to unravel subtle changes or relations in large datasets and to simplify the data. To this end, the intercorrelated variables of the data are transformed into principal components (PCs) which are orthogonal and linear combinations of the original variables. For further mathematical details we refer to literature. ${ }^{16}$

In this work, we present PCA of several IR-bands of DPPC and PHPC in monolayers, as measured with IRRAS. All spectra used in the PCA were measured in s-polarization at angles of incidence of the IR beam ranging from $25^{\circ}$ to $70^{\circ}$. Since the band shape of s-polarized IRRA spectra is independent of orientation of the absorbing group they can be used directly for PCA. ${ }^{17}$ The aim of this analysis is to correlate spectral changes with the phase state of the lipid and to obtain information about differences between both lipid species. We have chosen the spectral range of the carbonyl stretching vibrational band (1700 - $1775 \mathrm{~cm}^{-1}$ ) due to its sensitivity to hydration of the polarapolar interfacial region of the lipids. ${ }^{18}$

We measured IRRA spectra of the pure $\mathrm{H}_{2} \mathrm{O}$ subphase directly before collecting the monolayer spectra with identical measurement parameters. These water reference spectra were subtracted from each spectrum to reduce spectral contributions of the water vapor vibrational-rotational bands. The subtraction factor was determined by minimizing the variance of the second derivative of the spectrum in the spectral range of $3500-4000 \mathrm{~cm}^{-1}$. Since our aim was PCA of the carbonyl bands which overlap with the water deformation band $\gamma(\mathrm{H} 2 \mathrm{O})$, we additionally subtracted simulated water absorption bands of the measured monolayer to ensure minimal spectral contributions of the $\gamma(\mathrm{H} 2 \mathrm{O})$ band. Both subtractions were performed with home-written MATLAB scripts (MathWorks Inc., Natick, MA, USA).

Prior to PCA, a linear baseline was subtracted from the pretreated spectra and vector normalization of the carbonyl bands of all spectra were conducted. This ensures exclusion of major intensity differences between the bands from the PCs. The PCA was performed using the princomp function of MATLAB (MathWorks Inc., Natick, MA, USA).

Epifluorescence microscopy. As described previously, ${ }^{11}$ fluorescence images of monolayers being composed of DPPC, PHPC, and their mixtures, respectively, were recorded with an Axio Scope A1 Vario epifluorescence microscope (Carl Zeiss Microlmaging, Jena, Germany). Imaging was conducted during compression of the monolayer, which was performed as described before, allowing full control of the pressure status when the images were taken. A Teflon-coated trough $\left(26.6 \times 9.9 \mathrm{~cm}^{2}\right)$ equipped with a Wilhelmy balance (Riegler \& Kirstein, Potsdam, Germany) was mounted below the microscope on an $x-y-z$ stage (Märzhäuser, Wetzlar, Germany) which was controlled by a MAC5000 system (Ludl Electronic Products, Hawthorne, NY, USA). During measurements, a homebuilt Plexiglas hood covered the film balance. The microscope was equipped with a mercury short arc reflector lamp HXP 120 $C$, a long working distance objective (50x magnification, LD EC Epiplan-NEOFLUAR), and a filter/beam splitter combination which was appropriate for the used Rhodamine dye (all components from Carl Zeiss Microlmaging, Jena, Germany).

To measure fluorescence, 0.2 mol\% Rh-DPPE was added to the lipid solutions, before spreading the monolayers. This fluorescence dye partitions preferentially in lipid LE phases, which leads to a brightness contrast in phase separated monolayers. ${ }^{10,19}$

\section{Results and discussion}

The aim of this study is to compare monolayers of pure DPPC and PHPC, respectively, and to characterize their miscibility. First, we show how both lipids self-organize individually at the 
air-water interface using IRRAS parallel to measurements of the respective $\pi-A_{\mathrm{mol}}$ isotherms. Secondly, we present insights into mixed monolayers of DPPC and PHPC by measuring $\pi-A_{\mathrm{mol}}$ isotherms as a basis for subsequent thermodynamic analyses, and by performing epifluorescence measurements and IRRAS of the mixed monolayers.

\section{Monolayers of pure DPPC and pure PHPC}

To answer the question whether a small change in chemical structure-that is replacing one ester linkage between the glycerol and the $s n-2$ chain with an ether linkage-leads to measurable changes in lipid behaviour in monolayers, we performed $\pi-A_{\mathrm{mol}}$ isotherm measurements.

As can be seen in Figure 1, there are only small differences between $\pi-A_{\mathrm{mol}}$ isotherms of DPPC and PHPC. The only noteworthy differences are the slightly increased phase transition pressure of PHPC (4.42 $\mathrm{mN} \mathrm{m}^{-1}$ for DPPC vs. $6.05 \mathrm{mN} \mathrm{m}^{-1}$ for PHPC at $20^{\circ} \mathrm{C}$ ) and the overall higher compressibility of PHPC in the liquid-condensed (LC) phase compared to DPPC, as deduced from the decreased slope of the isotherm in the LC phase region. An increased transition pressure of PHPC, as measured in this work, is in accordance with literature reports ${ }^{8}$ while the difference in LC phase compressibility could be due to
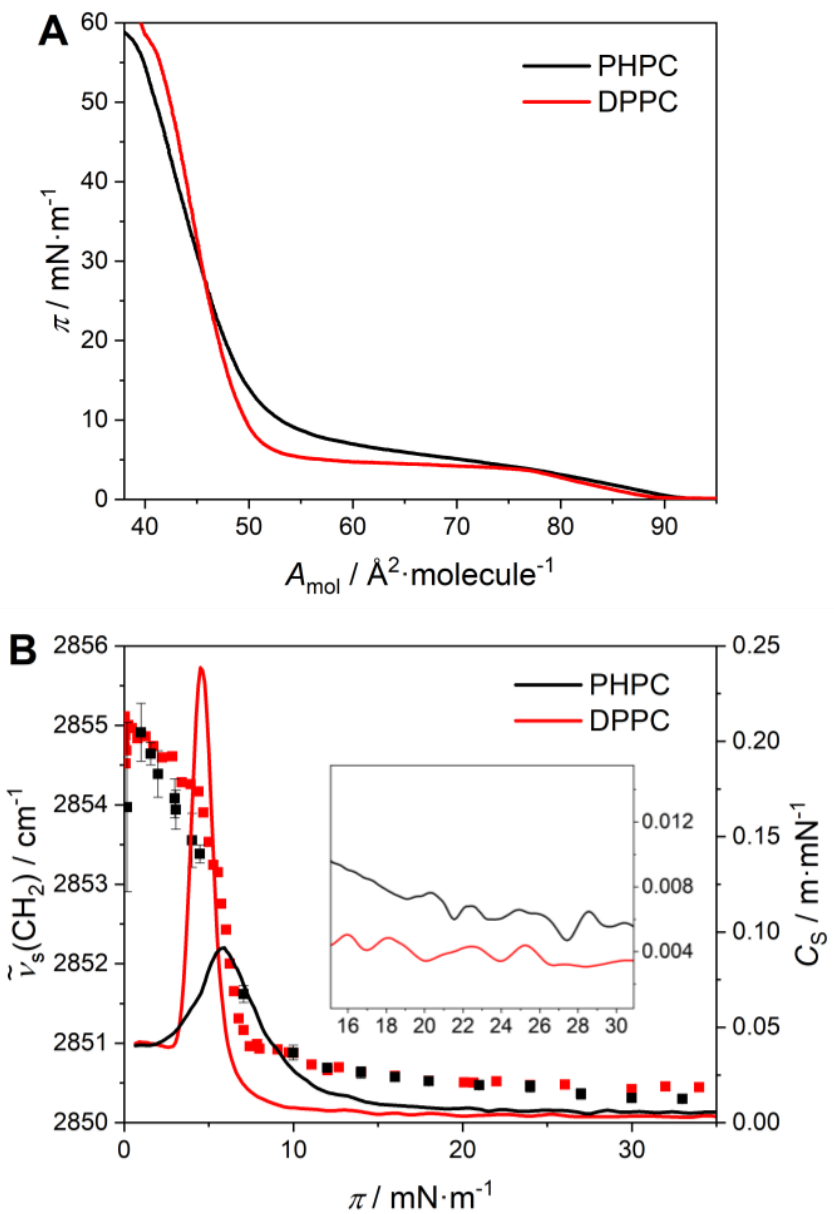

Figure 1. Langmuir isotherms and IRRAS data of pure DPPC and PHPC monolayers. A: $\pi-$ $A_{\text {mol }}$ isotherms of DPPC (red) and PHPC (black) at $20^{\circ} \mathrm{C} ; \mathrm{B}: v_{s}\left(\mathrm{CH}_{2}\right)$ frequency (scattered data) and compressibility (solid lines) of pure DPPC (red) and PHPC (black) monolayers at $20{ }^{\circ} \mathrm{C}$ plotted over surface pressure. The inset shows a magnification of $C_{S}$ in the LC phase (from 15 to $31 \mathrm{mN} \mathrm{m}^{-1}$ ). different pressure-dependent ordering of either lipid. A spectroscopic IRRA analysis was coupled to the isotherm measurements, with the aim to obtain more detailed insights into the organization of the lipids in the monolayer and to detect structural differences between DPPC and PHPC.

Both lipids were previously characterized in aqueous suspensions using IR spectroscopy. ${ }^{5,20}$ It was concluded that the main spectroscopic differences between both lipid bilayers are due to different orientations of the glycerol backbones. This effect seems to result in different hydration of the carbonyl moieties as well as conformational changes in adjacent methylene segments. ${ }^{5,} 20$ Furthermore, from X-ray diffraction data and $\pi-A_{\mathrm{mol}}$ isotherms of both lipids, Brezesinski et al. suggested different glycerol orientations in monolayers as well. ${ }^{6}$ In our study, we consequently first evaluated chain ordering and headgroup hydration in monolayers from IRRAS data.

For evaluating the order in the apolar lipid chain region, both symmetric and antisymmetric $\mathrm{CH}_{2}$ stretching vibrations were measured and simulated (see Figure S1 and Figure S2). Further details of the simulation procedure can be found elsewhere. ${ }^{13-15}$ The band position of both $\mathrm{CH}_{2}$ stretching vibrations are indicative of the trans/gauche ratio in alkyl chains. ${ }^{17,} 21$ Therefore, they are widely used to monitor phase transitions connected to chain melting. Simulation of angle- and polarization-dependent IRRA bands allows evaluating the order parameter $\mathrm{S}\left(\mathrm{CH}_{2}\right)$ of the whole all-trans chain if the orientation of the transition dipole moment is known. This order parameter can be translated into hydrocarbon chain tilt angle. The wavenumbers of $v_{s}\left(\mathrm{CH}_{2}\right)$ and $v_{\text {as }}\left(\mathrm{CH}_{2}\right)$ measured in this study are shown in Figure $1 \mathbf{B}\left(v_{s}\left(\mathrm{CH}_{2}\right)\right.$ as a function of surface pressure) and, additionally, in Figure $\mathbf{S 1}$ and Figure $\mathbf{S 2}$ together with the simulation results. The derived order parameters of the lipid chains as well as the resulting chain tilt angles in the LC phase are plotted in Figure $\mathbf{S 3}$ and included in Table 1. To interpret changes in $v_{s}\left(\mathrm{CH}_{2}\right)$ and, hence, changes in the trans/gauche ratio, we plotted $v_{s}\left(\mathrm{CH}_{2}\right)$ versus $\pi$ during monolayer compression in Figure 1B (scattered data). We only show the frequency of $v_{s}\left(\mathrm{CH}_{2}\right)$ because $v_{\text {as }}\left(\mathrm{CH}_{2}\right)$ contains overlapping contributions from $\mathrm{CH}_{3}$ group vibrations and a Fermi resonance band. ${ }^{22}$

\begin{tabular}{cccccc}
\hline \multicolumn{6}{c}{ Table 1. Summarized spectral parameters of pure DPPC and PHPC monolayers. } \\
\hline $\begin{array}{c}\pi / \mathrm{mN} \mathrm{m}^{-} \\
1\end{array}$ & $S\left(\mathrm{CH}_{2}\right)$ & $\theta /{ }^{\circ}$ & $\begin{array}{c}v_{\text {as }}\left(\mathrm{PO}_{2}^{-}\right) / \\
\mathrm{cm}^{-1 a}\end{array}$ & $S\left(\mathrm{PO}_{2}^{-}\right)^{\mathrm{b}}$ \\
\hline DPPC & 3 & 0.16 & $-^{\mathrm{c}}$ & 1225.2 & -0.18 \\
& 10 & 0.68 & 27.3 & 1226.0 & $-{ }^{\mathrm{d}}$ \\
& 20 & 0.71 & 26.1 & 1225.2 & -0.21 \\
PHPC & 30 & 0.73 & 25.3 & 1224.0 & -0.22 \\
& 3 & 0.05 & $-{ }^{c}$ & 1220.9 & -0.28 \\
& 10 & 0.68 & 27.5 & 1223.3 & -0.22 \\
& 20 & 0.75 & 24.0 & 1224.8 & -0.22 \\
& 30 & 0.84 & 19.4 & 1224.1 & -0.28 \\
\hline
\end{tabular}

a obtained by simulating the $v_{\text {as }}\left(\mathrm{PO}_{2}^{-}\right)$region including overlapping $\mathrm{CH}_{2}$ wagging band progressions

${ }^{b}$ simulated with the axis defined parallel to the transition dipole moment of $v_{\text {as }}\left(\mathrm{PO}_{2}^{-}\right)\left(\alpha=0^{\circ}\right)^{15}$

${ }^{c}$ not determined because lipid chains are not all-trans in the liquid-expanded (LE) phase

d it was not possible to fit the experimental phosphate band with $\alpha=0^{\circ}$ 
Our data suggest that the trans/gauche ratio of both lipids is similar since $v_{s}\left(\mathrm{CH}_{2}\right)$ are identical at similar $\pi$. We also found a comparably disordered LE phase in both lipid monolayers which becomes more ordered after phase transition to the LC phase. Directly after phase transition, at $10 \mathrm{mN} \mathrm{m}^{-1}, S\left(\mathrm{CH}_{2}\right)$ of both lipids are similar. However, during further compression, differences between DPPC and PHPC arise as PHPC forms more ordered monolayers above $20 \mathrm{mN} \mathrm{m}^{-1}$. In the LC phase, the chains of both lipids are in all-trans conformation as concluded from the $\mathrm{CH}_{2}$ stretching frequencies (see Figure 1B, scattered data). ${ }^{17,} 22$ Therefore, we calculated the tilt angle $\theta$ of the fully stretched lipid chains, which is also shown in Table 1. PHPC possesses a smaller tilt angle with respect to the surface normal than DPPC, which is in excellent accordance with x-ray data. ${ }^{6}$ In addition, $\theta$ of PHPC decreases further during LC phase compression as opposed to DPPC. This continuous film reorganization upon PHPC LC phase compression explains the higher compressibility of PHPC as compared to DPPC in the condensed phase as shown in Figure 1B (inset, solid lines).

Additional interpretation of deformation bands of the methylene groups is typically conducted to get insights into ordering effects of the chains and to elucidate coupling with other moieties. Evaluation of the $\mathrm{CH}_{2}$ scissoring vibrational band $\left(\delta\left(\mathrm{CH}_{2}\right)\right)$ enables us to detect the geometry of the lipid unit cell in the monolayer. ${ }^{17,}{ }^{21}$ For the two studied lipids, we found the frequency of the $\delta\left(\mathrm{CH}_{2}\right)=1468.9 \mathrm{~cm}^{-1}$ (at $30 \mathrm{mN} \mathrm{m}-1$ ) for both DPPC and PHPC in the LC phase (see Figure S4) while this band is not visible in the fluid LE phase. Both band positions in the LC phase are indicative of a hexagonal lattice which is in accordance with X-ray diffraction data. ${ }^{6,} 17$ During LC phase compression, the value slightly decreases, but no difference between both lipids could be detected. Another methylene deformation band, the $\mathrm{CH}_{2}$ wagging band progressions, will be discussed later in this work in combination with the antisymmetric phosphate vibration.

Headgroup vibrations of the characterized lipids contain information on ion binding and hydration of their polar moieties. Typical IRRA bands originating from headgroup vibrations of both lipids are the carbonyl stretching vibrational band $v(\mathrm{C}=\mathrm{O})$ and the antisymmetric phosphate stretching vibrational band $v_{\text {as }}\left(\mathrm{PO}_{2}^{-}\right)$. Both are shown in Figure 2. As can be seen directly, a small frequency decrease of the $v(C=O)$ band from DPPC to PHPC was found that is significantly less pronounced than the shift found in the bulk system. ${ }^{5,20}$ As opposed to aqueous suspensions, where complete subtraction of solvent spectra is possible, IRRA spectra inherently contain subphase contributions. Thus, the negative $v(C=O)$ overlaps with the positive water deformation band $\left(\gamma\left(\mathrm{H}_{2} \mathrm{O}\right)\right.$, see Figure $\left.2 \mathrm{~A}\right)$ which could hinder a direct band interpretation and shifts the $\mathrm{C}=\mathrm{O}$ band minima to higher wavenumbers. To circumvent this issue, we simulated the water absorption bands for each spectrum and subtracted them from the original data to yield spectra that are nearly free from water absorptions (see Experimental). Subsequently, we performed PCA of the corrected carbonyl bands to analyse a large dataset of spectra recorded at various pressures and angles for both lipids.
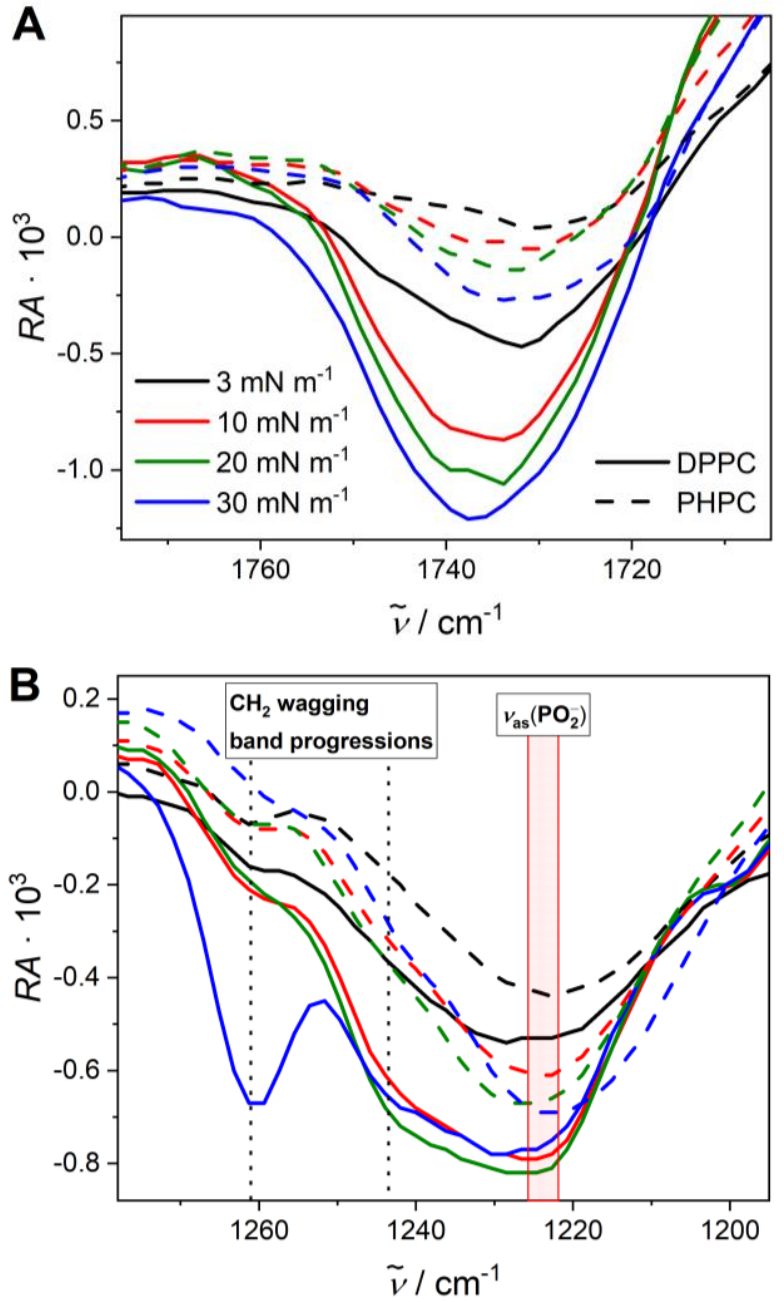

Figure 2. Headgroup vibrations of pure PHPC (dashed lines) and DPPC (solid lines) monolayers at $20^{\circ} \mathrm{C}$ and at different surface pressure as indicated. $\mathbf{A}: v(\mathrm{C}=0)$ band, $\mathbf{B}$ $v$ as $\left(\mathrm{PO}_{2}^{-}\right)$region including $\mathrm{CH}_{2}$ wagging band progressions as indicated.

The frequency of $v(C=O)$ can be interpreted in terms of hydration of the carbonyl group. ${ }^{5,18,20}$ In general, increasing hydration shifts the band centre to lower wavenumbers. However, it is not possible to derive the number of bound water molecules directly without knowing the exact absorption coefficients of the dehydrated, monohydrated, and dihydrated species, respectively. In addition, hydrogen bonding by more water molecules and stronger hydrogen bonding are indiscernible from IRRAS frequency shifts. ${ }^{23}$ Still, in aqueous suspensions, different subcomponents of the $v(C=O)$ band are interpreted as different hydration states of the carbonyl group containing distinct numbers of bound water molecules. ${ }^{18}$ The spectra shown in Figure $\mathbf{2 A}$ are therefore indicative of differences in hydration of the headgroups between both lipids. However, a more precise analysis is necessary due to different influence of the overlapping $\gamma\left(\mathrm{H}_{2} \mathrm{O}\right)$ band on the $\mathrm{C}=\mathrm{O}$ bands of both lipids. In contrast to IR measurements of aqueous suspensions, we are not able to detect whether two or three subcomponents ${ }^{20}$ are included in the lipids' carbonyl stretching vibrational band because of the overlap of the $v(C=O)$ band with the water deformation band. 

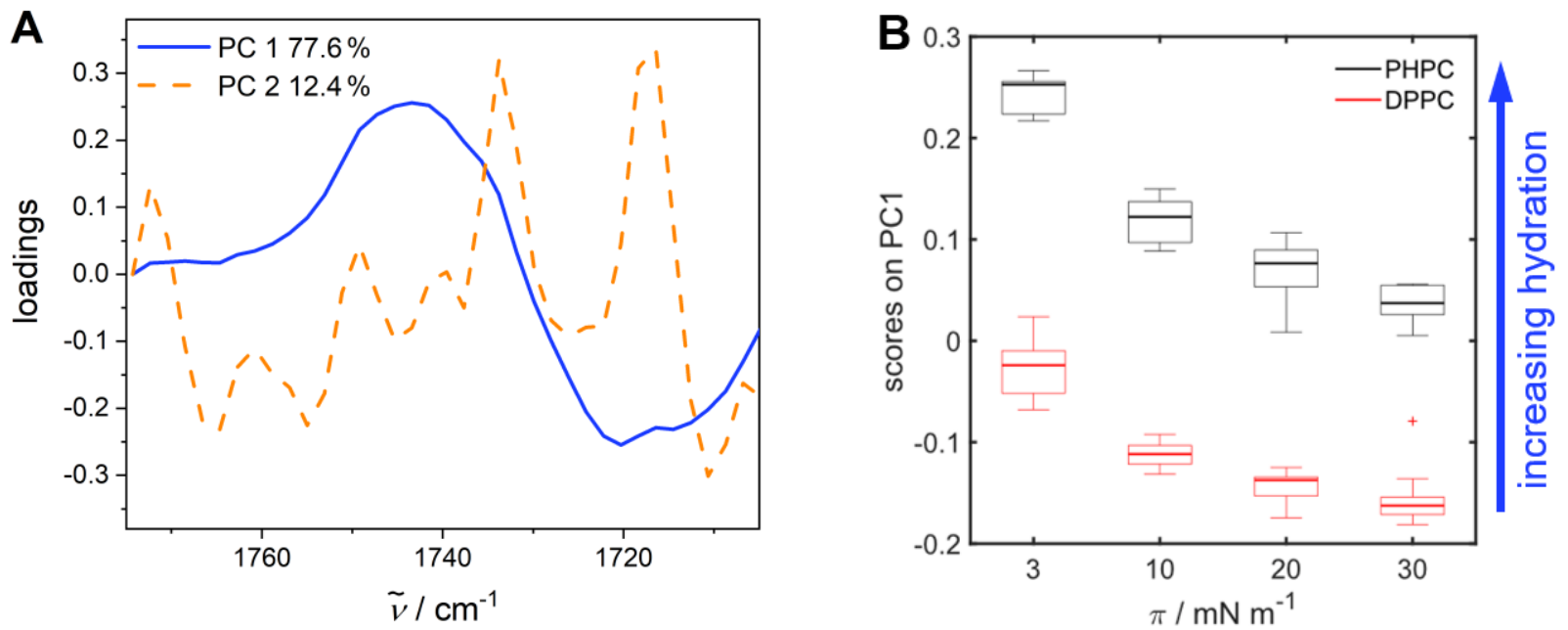

Figure 3. PCA results of the $v(C=0)$ band of pure PHPC and DPPC monolayers at different surface pressures. A: loadings of PC 1 (blue) and PC 2 (orange); $\mathbf{B}$ : score on PC 1 versus surface pressure $\pi$ shown as box-whisker-plot. The IRRAS data used in this PCA were measured in the LE phase of both lipids $\left(3 \mathrm{mN} \mathrm{m}^{-1}\right)$ and in the LC phase $\left(10 \mathrm{mN} \mathrm{m}^{-1}, 20 \mathrm{mN} \mathrm{m}^{-}\right.$ 1 , and $30 \mathrm{mN} \mathrm{m}^{-1}$ ) of DPPC (red) and PHPC (black), respectively. The (+) at $30 \mathrm{mN} \mathrm{m}^{-1}$ refers to an outlier for DPPC.

Using IRRAS, we evaluated the $v(C=O)$ bands of both pure lipid monolayers at four distinct surface pressures, namely at $3 \mathrm{mN} \mathrm{m}^{-1}$ in the LE phase and at 10,20 , and $30 \mathrm{mN} \mathrm{m}^{-1}$ in the LC phase. For each surface pressure, we performed angle- and polarization-dependent IRRA measurements that allow fitting the data to unravel conformational differences between both lipids and subtraction of the water absorption bands, which only depend on monolayer thickness, monolayer refractive index, and the quality of the used polarizer. The latter was determined empirically from all measurements with the used polarizer to be $\Gamma=0.007$. The monolayer refractive index of phospholipids is known from literature $(n=1.41) .{ }^{24}$ Monolayer thickness as the only remaining parameter can be derived from fitting the theoretical subphase water absorption bands to the measured data. After subtracting the simulated water bands from all IRRA spectra, we conducted a PCA of the vector-normalized carbonyl bands of all s-polarized spectra of both lipids recorded at various angles of incidence $\left(25-70^{\circ}\right)$ and surface pressures. The results of the PCA are shown in Figure 3 and Figure S5/S6.

The interpretation of spectral PCA is not necessarily straightforward. At first, one must interpret the resulting loadings of the principal components (PCS). PC 1 and PC 2 (Figure 3A) account for approximately $90 \%$ of all differences in the $v(C=O)$ dataset and, hence, we limit our interpretation to these PCs. The loading of PC 1 reflects a spectral shift of the band minima from high to low frequency. Thus, higher scores on PC 1 are indicative for a higher hydration of the interfacial carbonyls, which is not visible as clearly in the averaged spectra without PCA (compare to Figure 2A). The second PC, in contrast, seems to result mainly from atmospheric water vapor and experimental noise. It is an advantage of the PCA that these disturbing contributions are separated from the systematic variations mapped through PC 1 . To interpret the differences between DPPC and PHPC, the score of PC 1 versus the surface pressure is shown in Figure 3B as a box plot, were the individual boxes contain contributions of IRRA spectra measured at various angles of incidence. Figure 3B shows a clear separation of the scores of DPPC and PHPC on the PC 1 axis at all examined surface pressures, indicating different hydration of their respective carbonyl moieties. Additionally, within each subset of data, the scores on the PC 1 decrease as well with increasing compression of the monolayer. For both lipids, this shift is most pronounced between 3 and $10 \mathrm{mN} \mathrm{m}^{-1}$, corresponding to the LE/LC phase transition. Within the LC phase, this shift is smaller; however, it is more pronounced for PHPC than for DPPC. This reflects once more the ongoing ordering of PHPC upon LC phase compression corresponding to the chain order parameter and compressibility, respectively, reported above.

The scores of PC 2 versus PC 1 are shown in Figure S5. No systematic changes are visible in the second $P C$, which matches the assumption of stochastic causes for the PC 2 . The angle of incidence did not affect the score of any spectrum on the PC 1 as can be seen in Figure $\mathbf{S 6}$. However, the score of PC 2 depends on the angle of incidence, which, in turn, is due to the dependence of surface reflectivity on $\varphi$. This means the reflectivity and, hence, the signal-to-noise ratio increases for spolarized IR light with increasing angle of incidence. ${ }^{17}$

As can be interpreted from Figure 3, the PCA yields two results:

a) at all surface pressures, the frequency of the $v(C=O)$ band of PHPC is decreased compared to DPPC and

b) for both lipids, the frequency of the $v(\mathrm{C}=\mathrm{O})$ band increases upon compression.

Since a frequency difference of the carbonyl stretching vibration between DPPC and PHPC reflects different hydration of the carbonyl group, we conclude that the carbonyl moiety of PHPC is either more hydrated (more bound water molecules) or the existing water molecules are bound more tightly to the carbonyls at the interface when compared to DPPC. This finding at first glance seems counterintuitive, as the substitution of an ester linkage between the glycerol and the sn-2 chain with an ether should result in a slightly more apolar headgroup region in comparison to DPPC. However, this seems not to decrease the hydration of the remaining carbonyl, but rather increases it. 
With respect to the literature, this may be interpreted as a change in glycerol orientation from approximately perpendicular to the water surface in DPPC to parallel to the water surface in PHPC-similar to findings in aqueous (bulk) suspensions. ${ }^{5}$ An altered glycerol orientation in the gel phase of PHPC in bulk is connected to the formation of an interdigitated gel phase $\left(L_{\beta} \mid\right)$. In contrast, in monolayers an interdigitated arrangement is obviously not possible.

To support this hypothesis, we also evaluated the $\mathrm{CH}_{2}$ wagging band progressions $\left(1260-1262\right.$ and $\left.1243 \mathrm{~cm}^{-1}\right)$ of both lipids in monolayers, which overlap with the antisymmetric phosphate stretching vibrational band, $v_{\text {as }}\left(\mathrm{PO}_{2}{ }^{-}\right)$(1221 $1226 \mathrm{~cm}^{-1}$ ), of the headgroup. ${ }^{25}$ The $\mathrm{CH}_{2}$ wagging band progressions are sensitive to single gauche conformers near the carbonyl groups of the lipid in the LC phase, because the intensity of the wagging band progressions increases significantly with coupling to the carbonyl group. ${ }^{22}$ Adjacent kinks within the alkyl chain, i.e. the presence of gauche conformers, prevent this coupling resulting in attenuation of the $\mathrm{CH}_{2}$ wagging band progressions. No band progressions of the $\mathrm{CH}_{2}$ wagging vibration are therefore observed in fluid $\mathrm{LE}$ phases. ${ }^{22}$ When comparing PHPC with DPPC, this effect is superimposed by the attenuation of these bands due to removal of one carbonyl group in PHPC. However, in DPPC bilayers, the $\mathrm{CH}_{2}$ wagging band progressions almost exclusively originate from the $s n-1$ chain, since the $s n-2$ chain includes a kink adjacent to the carbonyl group. ${ }^{5}$ Therefore, if PHPC adopts a similar conformation of the glycerol backbone and the $s n-1$ ester linkage compared to DPPC, no or only little attenuation should occur. However, the $\mathrm{CH}_{2}$ wagging band progressions are significantly attenuated in bulk, which was considered by Lewis et al. as an additional argument for different glycerol orientations when comparing DPPC and PHPC. ${ }^{5}$

Our results in monolayers, as shown in Figure 2B, expose an almost complete vanishing of the $\mathrm{CH}_{2}$ wagging band progressions of PHPC as compared to DPPC independent of the lipid phase. This must be caused by the introduction of one or more gauche conformers adjacent to the carbonyl moiety of the $s n-1$ chain. ${ }^{22}$ Since the glycerol orientation in the LC phase of typical diester phosphocholines does not induce this gauche conformer ${ }^{26}$ and measured spectra, thus, show significant coupling of carbonyl group and $\mathrm{CH}_{2}$ wagging vibrations, ${ }^{5}$ we interpret this spectral difference of PHPC again in terms of a different glycerol orientation. By comparing our monolayer studies with literature-based knowledge from aqueous suspensions, ${ }^{5}$ it becomes obvious that the attenuation of the $\mathrm{CH}_{2}$ wagging band progressions is remarkably more pronounced in monolayers than in bilayers. This can be interpreted as the $s n-1$ carbonyl moieties and the $s n-1$ chains having different orientations towards each other in bulk and in the monolayer, respectively. Additionally, all molecules in the monolayer are probably more uniformly arranged when compared to the bulk system, i.e., one adjacent gauche conformer is introduced in all molecules in the PHPC monolayer. ${ }^{22}$ However, these differences are expected because the typical arrangement of lipid molecules in monolayer LC phases is by no means the same as in interdigitated gel phases in bulk. It is remarkable that a change as small as substitution of one carbonyl with a $\mathrm{CH}_{2}$ group has a similar effect on the glycerol backbone conformation as changing the whole chain position from $s n-2$ to $s n-3$ and, thus, forcing the glycerol to be oriented parallel to the bilayer surface, as found in 1,3-dipalmitoylglycerophosphocholine (1,3-DPPC, $\beta$ DPPC $)^{27,} 28$ and 1,3-diamidophospholipids, respectively. ${ }^{29,} 30$

The absence of $\mathrm{CH}_{2}$ wagging band progressions in the $\mathrm{LE}$ phase of both lipid monolayers allows direct comparison of the $v_{\text {as }}\left(\mathrm{PO}_{2}^{-}\right)$bands. This band of the polar headgroup is sensitive to ion binding as well as hydrogen bonding. ${ }^{17,}{ }^{21}$ Since we used deionized water for all experiments, we may neglect contributions from remaining ions. Consequently, frequency shifts of $v_{a s}\left(\mathrm{PO}_{2}^{-}\right)$are caused by hydrogen bonding to either water or other lipid molecules. However, the phosphate groups in phosphocholines are known to be proton acceptors while no acidic protons can be donated from phosphocholines (unlike, e.g., phosphoethanolamines). ${ }^{31}$ Thus, hydrogen bonds cannot be formed directly with other lipids and must always include hydrating water molecules. In the IRRA spectra of both lipids at $3 \mathrm{mN} \mathrm{m}{ }^{-1}, \mathrm{CH}_{2}$ wagging band progressions are absent as expected, therefore, the frequency of the $v_{a s}\left(\mathrm{PO}_{2}^{-}\right)$band could be derived by simulating the spectra using only a single component. The results are summarized in Table 1. From the data it becomes evident that PHPC exhibits a lower $v_{a s}\left(\mathrm{PO}_{2}^{-}\right)$ frequency than DPPC. Similar to what we found for the carbonyl stretching vibrational band, this again indicates an increased hydration of the PHPC headgroups, i.e. more or stronger bound water molecules. ${ }^{31,32}$

By simulating the $v_{\text {as }}\left(\mathrm{PO}_{2}^{-}\right)$region including $\mathrm{CH}_{2}$ wagging band progression contributions in the LC phase, it is possible to calculate the spectral components of these overlapping bands directly (see selected data in Figure 4, all spectra in Figure S7 and Figure S8). For simulation, we used literature values for $\mathrm{CH}_{2}$ wagging band progression frequencies of DPPC in aqueous suspensions as starting parameters ${ }^{25}$ and adjusted them in a nonlinear least square fit. However, the frequencies fitted to our measurements did not deviate more than $5 \mathrm{~cm}^{-1}$ from literature values despite being measured in LC phase monolayers instead of gel phase bilayers. Especially the evaluation of the order parameter $S\left(\mathrm{PO}_{2}^{-}\right)$from $v_{\text {as }}\left(\mathrm{PO}_{2}^{-}\right)$is promising as it can be correlated with the headgroup ordering. The determined order parameters of the $v_{\text {as }}\left(\mathrm{PO}_{2}{ }^{-}\right)$are shown in Figure $\mathbf{5 9}$ and Table 1.

We simulated $S\left(\mathrm{PO}_{2}^{-}\right)$with respect to the axis defined by the transition dipole moment of $v_{\text {as }}\left(\mathrm{PO}_{2}^{-}\right)\left(\alpha=0^{\circ}\right)^{15}$ to evaluate only the orientation of the phosphate group itself. $S\left(\mathrm{PO}_{2}^{-}\right)$of both lipids depends on the lipid phase and our data clearly show differences between DPPC and PHPC. The phosphate groups of DPPC monolayers are less ordered, i.e., $S\left(\mathrm{PO}_{2}^{-}\right)$is smaller when compared to PHPC, regardless of the monolayer phase state. While DPPC maintains similar $S\left(\mathrm{PO}_{2}^{-}\right)$values over the whole compression range, $\mathrm{PHPC}$ shows a jump during phase transition from a higher magnitude of $S\left(\mathrm{PO}_{2}^{-}\right)$in the LE phase to a lower magnitude of $S\left(\mathrm{PO}_{2}^{-}\right)$in the LC phase. Beyond the $\mathrm{LE} / \mathrm{LC}$ phase transition, both lipids exhibit a decreasing $S\left(\mathrm{PO}_{2}^{-}\right)$during further compression of the condensed monolayer, with PHPC covering a larger range of ordering. As can be seen in Figure $\mathbf{S 9}, \mathrm{S}\left(\mathrm{PO}_{2}{ }^{-}\right)$ of PHPC decreases significantly during compression of the LC 

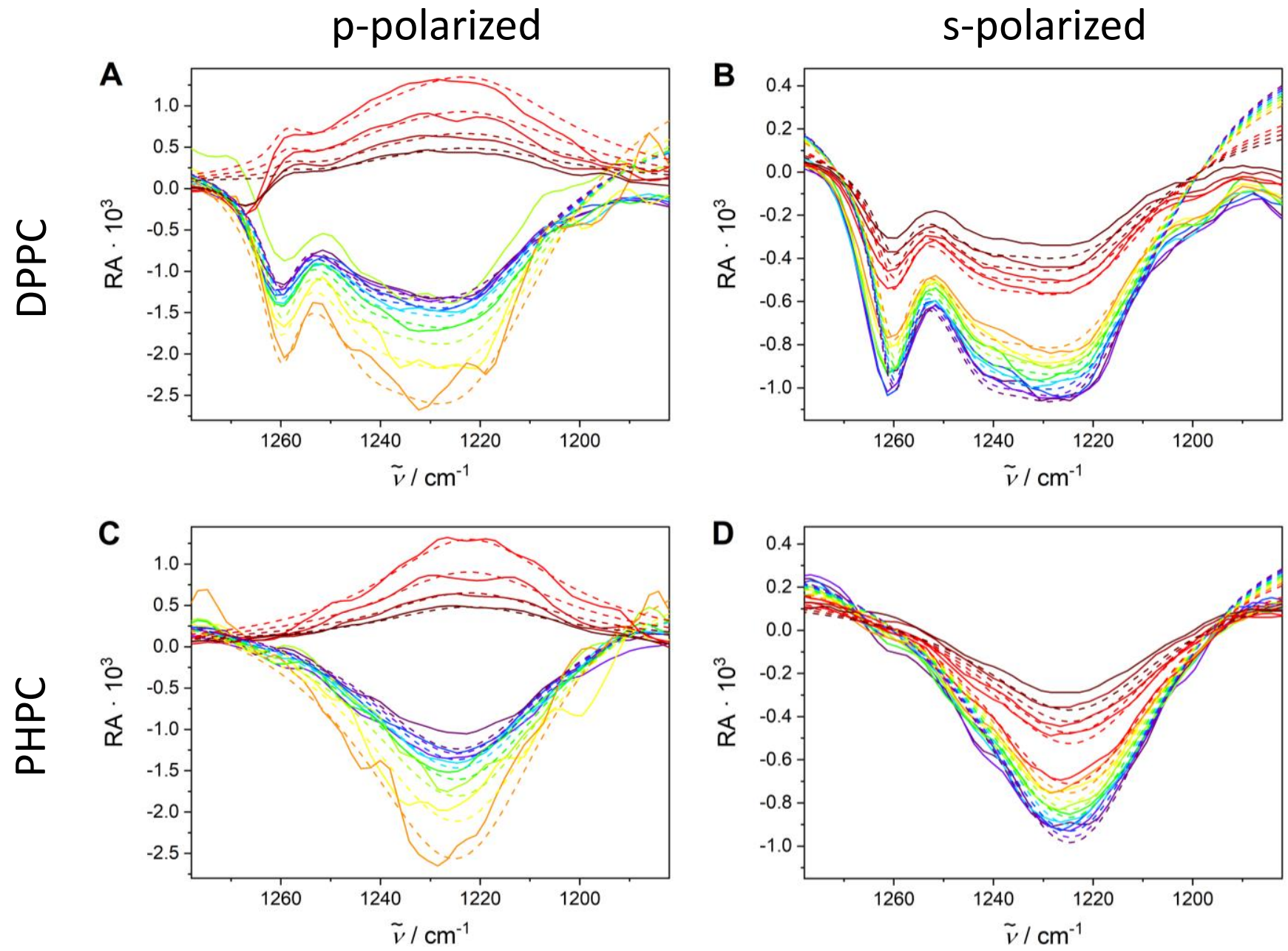

Figure 4. Selected fits of the antisymmetric phosphate region including $\mathrm{CH}_{2}$ wagging band progressions at $30 \mathrm{mN} \mathrm{m}{ }^{-1}$ in the $\mathrm{LC}$ phase of pure DPPC (A, p-polarized; $\mathbf{B}$, s-polarized) and PHPC (C, p-polarized; D, s-polarized).

phase. However, this effect should be interpreted cautiously as only three order parameters were measured in this lipid phase.

All the observed differences between DPPC and PHPC regarding their $S\left(\mathrm{PO}_{2}^{-}\right)$are associated with the different glycerol orientation of both lipids, which must be caused by the comparably small chemical change of the sn-2 linkage.

With respect to all the results regarding the phosphate group, we conclude that an increased hydration of the PHPC phosphate in the LE phase goes along with a higher degree of headgroup ordering. These effects do not occur in DPPC monolayers and may therefore be related to different arrangements of glycerol backbones when DPPC and PHPC are compared. Presumably, more or strongly bound water molecules increase the ordering of the phosphate moiety in PHPC monolayers. Furthermore, while in DPPC monolayers significant overlapping of $\mathrm{CH}_{2}$ wagging band progressions with $v_{\text {as }}\left(\mathrm{PO}_{2}^{-}\right)$ impede interpretation of separate bands, the corresponding bands in PHPC are attenuated to such a degree that they are hardly observable at all. This, firstly, supports our interpretation of a glycerol backbone oriented parallel to the surface and, secondly, allows direct interpretation of the $v_{\text {as }}\left(\mathrm{PO}_{2}^{-}\right)$band.
In summary, the substitution of the $s n-2$ ester bond in DPPC by an ether bond in PHPC leads to:

- a different ordering of the lipid alkyl/acyl chains while maintaining the overall phase behaviour,

- a rearrangement of the glycerol backbone to a presumably parallel orientation with respect to the water surface connected with the introduction of (at least one) gauche conformers in the $s n-1$ alkyl chain adjacent to the carbonyl moiety, and

- a higher degree of headgroup ordering combined with increased hydration of both the carbonyl and the phosphate group.

The resulting different molecular orientations of DPPC and PHPC molecules at the air-water interface are schematically depicted in Scheme 1.

\section{Mixed monolayers of DPPC and PHPC}

In the second part of this study, we focus on the mixing behaviour of DPPC and PHPC in monolayers at the air-water interface. For this purpose, we measured $\pi-A_{\text {mol }}$ isotherms of mixed monolayers containing both lipids at different mixing ratios at $20^{\circ} \mathrm{C}$ and, simultaneously, performed epifluorescence 


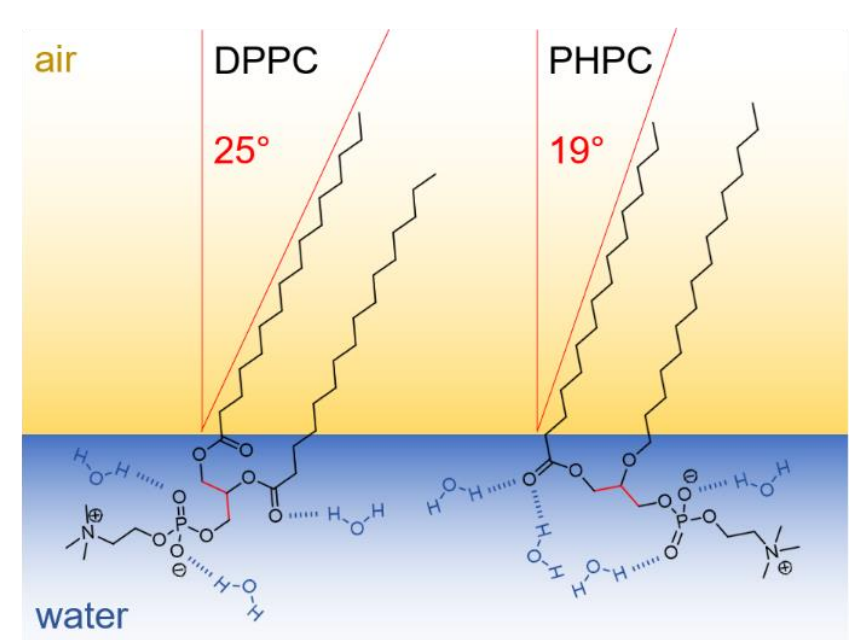

Scheme 1. Schematic representation of a possible LC phase orientation of a DPPC and a PHPC molecule on the air-water interface as deduced from IRRA spectroscopy. The number and orientation of the water molecules and hydrogen bonds, respectively, are schematic and for illustration purposes only.

microscopy of these mixed monolayers. Furthermore, we measured IRRA spectra of the corresponding mixtures of PHPC and DPPC- $d_{62}$ which is the chain-perdeuterated analogue of DPPC, to obtain spectral selectivity between the two lipids.

The $\pi-A_{\mathrm{mol}}$ isotherms of mixtures containing 90 to $25 \%$ DPPC and 10 to $75 \%$ PHPC are shown in Figure 5A together with the isotherms of the pure compounds. In the isotherms of the mixtures, two plateaus or kinks are observable, the upper of which does not exist in isotherms of the pure substances. Therefore, the question arises, whether this plateau is connected to a phase transition or not. This issue can only be elucidated using additional techniques and will be discussed later. By evaluating the compressibility maxima (see Figure S10) of all films, the surface pressure values at both plateaus were determined. These values are plotted in Figure 5B in form of a partial phase diagram and are further shown in Table 2 . The midpoint of the lower transition is always located at the transition pressure of pure PHPC, whereas the upper plateau pressure increases with increasing PHPC content, which also has to be discussed in terms of phase transitions, below.

Table 2. Plateau surface pressures ( $\pi_{\text {plateau }}$ ) of pure and mixed monolayers of PHPC and DPPC.

\begin{tabular}{ccr}
$x(\mathrm{PHPC})$ & \multicolumn{2}{c}{$\pi_{\text {plateau }} / \mathrm{mN} \mathrm{m}^{-1}$} \\
\hline 0.00 & \multicolumn{2}{c}{$4.42 \pm 0.23$} \\
0.10 & $6.12 \pm 0.12$ & $11.48 \pm 0.38$ \\
0.25 & $6.20 \pm 0.13$ & $12.25 \pm 0.35$ \\
0.33 & $5.83 \pm 0.19$ & $13.22 \pm 0.14$ \\
0.50 & $5.97 \pm 0.15$ & $13.87 \pm 0.28$ \\
0.67 & $5.92 \pm 0.24$ & $15.33 \pm 0.01$ \\
0.75 & $6.11 \pm 0.08$ & $15.64 \pm 0.19$ \\
1.00 & \multicolumn{2}{c}{$6.05 \pm 0.15$} \\
\hline
\end{tabular}

To further characterize the phase behaviour of the mixed monolayers, epifluorescence microscopy was performed using Rh-DPPE as fluorescent dye. The obtained micrographs are shown in Figure 6 (including pure DPPC $(x($ PHPC) $=0)$ ), top row, and pure PHPC $(x(\mathrm{PHPC})=1)$, bottom row, monolayers $)$ and
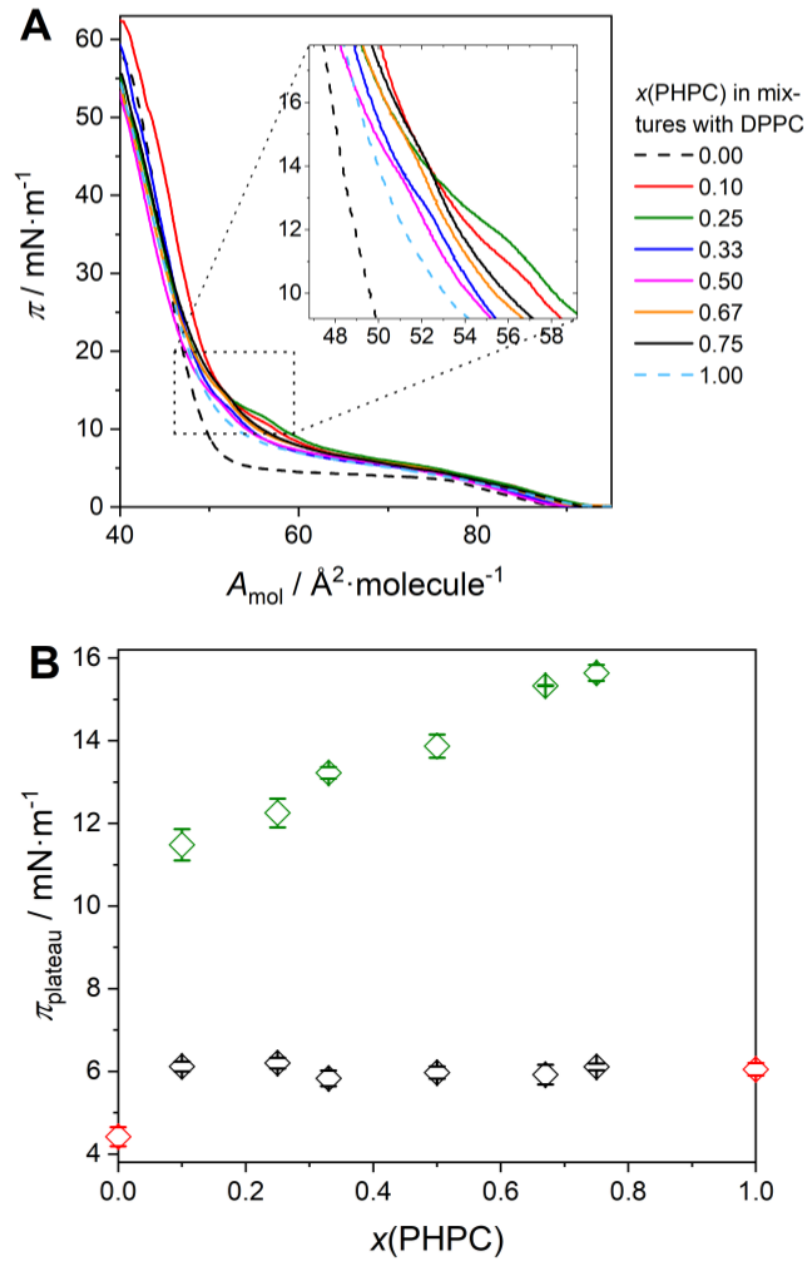

Figure 5. Isothermal compression of mixed DPPC/PHPC monolayers at $20^{\circ} \mathrm{C}$. A: $\pi-A_{\text {mo }}$ isotherms with $\pi$ range of the upper plateau in the inset, B: phase diagram with all observed $\pi_{\text {plateau }}$ (pure lipids are marked in red, $\pi$ plateau of the upper plateau is highlighted green)

additionally in Figure S11-S16. Typically, Rh-DPPE dissolves readily in LE-phase monolayers while it is excluded from LCphase domains. ${ }^{10,19}$ As can be seen in column (c) of Figure 6, this is true for a pure DPPC monolayer, where LC domains appear black. ${ }^{19}$ In contrast, in PHPC-containing monolayers, RhDPPE can partition into LC domains leading to a lower contrast between the LE and LC phase. Moreover, the shape of DPPC and PHPC LC domains is remarkably different. Therefore, changes in LC phase composition can be unravelled by contrast and shape of the observed domains in mixed monolayers of DPPC and PHPC.

First, epifluorescence microscopy of the mixed monolayers enables us to determine, whether (partial) demixing occurs in the LE and/or the LC phase of the mixed monolayers. As it is evident from Figure 6 and Figure S12-S15, the LE phases of all measured mixtures are uniformly bright. This leads to the conclusion that no phase separation occurs below the lower plateau of the respective mixture. At surface pressures above this plateau, the micrographs of all DPPC/PHPC mixtures appear uniformly grey indicating the existence of only one homogeneously mixed phase (column (e) in Figure 6). No further changes were detected at the upper plateau of the compression isotherms. 
Figure 6. Selected fluorescence micrographs of mixed monolayers of DPPC and PHPC. The scale bars represent $20 \mu \mathrm{m}$ in case of DPPC and $50 \mu \mathrm{m}$ in all other micrographs. The micrographs shown here were taken in the LE phase, at different points in the LE/LC transition region as indicated in the column headings, and in the LC phase. A full picture of the fluorescence microscopic characterization is presented in Figure S11-S16. 
Second, the LE/LC transition of the mixtures, which evidently occurs at the lower plateau, can be studied in comparison to the LE/LC transition of both pure substances. The LC domain shape of pure DPPC and PHPC differs significantly (see Figure 6). While DPPC forms characteristic chiral bean or propeller shaped domains, PHPC, which is racemic in this study, forms star-like, fractal grey LC domains which do not exhibit chirality. ${ }^{33}$ When both lipids are mixed, nucleation of the LC domains begins with mainly DPPC, as can be deduced from the appearance of compact black domains (see column (b) in Figure 6). Rh-DPPE is excluded from these small DPPC-rich domains/ nuclei at the onset of the LE/LC transition but, subsequently, partitions into the LC phase while PHPC is incorporated. As the LC domains grow in size, PHPC joins at the rim of already formed DPPC domains with its typical star-like LC domain shape leading to demixed LC domains (see column (c) in Figure 6). At the end of the phase transition, the LC phase homogenizes, i.e., both lipids form a mixed LC phase, being embedded in a continuous LE phase (see column (d) in Figure 6). Mixtures with high DPPC content remain in the demixed LC state up to higher surface pressures and their LC phases tend to be darker because the dye is excluded from DPPC-rich domains to a higher extent. Although these results suggest a co-existence of three phases in the lower plateau, these three phases were not stable when the compression was paused (see Figure S17). Likewise, only two phases could be observed in the LC/LE transition upon expansion of a mixed DPPC/PHPC monolayer $(x(\mathrm{PHPC})=0.1)$ from the $L C$ phase (see Figure S17). We therefore assume that the three phases occurring in the LE/LC transition region upon compression are meta-stable and not in equilibrium, i.e., their appearance is due to a kinetically hindered condensation upon continuous compression.

Yet, the origin of the upper plateau remains uncertain. By means of fluorescence microscopy and with the used Rh-DPPE dye it could not be attributed to a phase transition. To unravel further details of lipid miscibility, we interpret our data by applying the Gibbs phase rule and by calculating the Gibbs energy of mixing, ${ }^{10}$ both of which are regularly used in miscibility studies in monolayers.

Although the surface phase rule is frequently used for miscibility studies in Langmuir monolayers, 9,34 in this work application of the Gibbs phase rule is sufficient as shown in Appendix 1 . The Gibbs phase rule is given as $F=C-P+2$, where $F$ denotes the degrees of freedom, $C$ is the number of components in the monolayer, and $P$ is the number of phases in the monolayer. It is possible to only discuss the components and phases of the monolayer as long as no lipid exchange between monolayer and bulk phases occurs, which is the case for longchain phospholipids. ${ }^{35}$ With two monolayer components, DPPC and PHPC, the Gibbs phase rule simplifies to $F=4-P$ or $P=$ $4-F$, when solved for the number of phases. Thus, by evaluating the degrees of freedom of the studied systems, a prediction of the number of co-existing phases is possible. Since there must be at least one monolayer phase, maximal three degrees of freedom can exist, which are $\pi, T$ and $x$. Consequently, $P$ can vary between one $(F=3)$ and four $(F=0)$.
As evident from Figure 5 , in all phases between the $\pi_{\text {plateau }}$ as well as above and below them, $\pi$ and $x$ and presumably $T$ (compare Figure S18) are degrees of freedom, as they can be varied independently without changing the state of the system. Hence, only one mixed phase would exist in these states. This is consistent with the results of epifluorescence microscopy which show one uniform phase in the respective surface pressure ranges (Figure 6, columns (a) and (e) and Figure S12-S15) as well as the analysis of $\Delta G_{\text {mix }}$ discussed below.

In contrast, in the plateaus of the $\pi-A_{\mathrm{mol}}$ isotherms, $x$ and $\pi$ are correlated (Figure 5B), i.e., $\pi$ depends on $x$ and is therefore not a degree of freedom as opposed to $x$. Consequently, $F$ is either one $(x)$ or two $(T, x)$ and, hence, $P$ is three or two, respectively. Note that $\pi$ of the lower plateau seems to be independent of $x$ due to both pure lipids exhibiting nearly the same LE/LC transition pressure. However, if chain-perdeuterated DPPC- $d_{62}$ is used in these mixtures is, which has an increased phase transition pressure, ${ }^{36,37} \pi$ of the lower plateau also depends on $x$ (see below and Figure S20). Interestingly, the epifluorescence micrographs shown in columns (c) and (d) of Figure 6 and further in Figure S12-S15 display three apparent phases in the lower plateau of the mixtures, which are not stable (see Figure S17), and are probably the consequence of the continuous compression of the monolayer. Hence, they do not represent the equilibrium state within the LE/LC phase transition and, consequently, cannot be interpreted using the phase rule which only is valid in equilibrium. This means that the number of equilibrium phases in the transition is two and consequently both $x$ and $T$ are degrees of freedom, i.e., $\pi$ depends on $x$ and on $T$. The latter, $\pi$ depends on $T$, was proven exemplarily for one mixture (Figure S18). One can clearly see that the compressibility maxima of the DPPC/PHPC $3: 1$ monolayer shift linearly to lower surface pressure with decreasing temperature. This shows that the transition exists at different temperatures and, hence, $T$ is a degree of freedom in the phase transition. This allows the conclusion that beyond the plateaus no phase coexistence occurs.

To further evaluate the thermodynamics of the miscibility of both lipids below, in between, and above the plateaus, one can calculate the Gibbs energy of mixing $\Delta G_{\text {mix }}$ and the excess Gibbs energy of mixing $\Delta G_{\text {exc }}$, which describes the deviations from ideal miscibility. ${ }^{10}$ To this end, we compare the observed $\pi-A_{\text {mol }}$ isotherms with ideal ones, calculated from the pure substances, to yield molecular excess areas $A_{\text {exc }}$. The $A_{\text {exc }}$ are then integrated and values of $\Delta G_{\text {exc }}$ are calculated. Using the known ideal Gibbs energy of mixing, $\Delta G_{\text {mix }}$ is then derived. Further information on calculations are given in the Experimental part of this study. The calculated $\Delta G_{\text {exc }}$ and $\Delta G_{\text {mix }}$ are included in Table $\mathbf{S 1}$ and plotted in Figure 7.

From these data it can be concluded that at all mixing ratios, DPPC and PHPC are miscible. In the LE phase at $3 \mathrm{mN} \mathrm{m}^{-1}$, nearly ideal mixing or complete demixing was observed. As stated before, a complete immiscibility in the LE is rather unlikely and was not found using epifluorescence microscopy. Therefore, we interpret this result as ideal miscibility of both lipid species in the LE phase. However, positive deviations from an ideal miscibility at $10 \mathrm{mN} \mathrm{m}^{-1}$ (between both plateaus) and $30 \mathrm{mN} \mathrm{m}^{-1}$ 
(above both plateaus), i.e. repulsive interactions between both lipids, were observed for the DPPC/PHPC mixtures consisting of $10 \%$ and $75 \%$ of PHPC, respectively (Figure $7 \mathrm{~A}$ ). However, $\Delta G_{\text {exc }}$ is not high enough to induce a complete phase separation.

To evaluate the miscibility in more molecular detail, again infrared reflection absorption spectroscopy (IRRAS) was used. IRRAS enables us to discriminate both lipids in mixtures through isotopic labelling, since different nuclear isotopes result in different reduced masses of the vibrating moieties. Hence, a shift is observed in the IR spectra. When mixing PHPC with chain-perdeuterated DPPC- $d_{62}$ it is, thus, possible to compare the surface pressure-dependent change in frequency of $v_{s}\left(\mathrm{CH}_{2}\right)$ and $v_{s}\left(C_{2}\right)$, to observe the phase transition pressure of both lipids in their mixtures independently. If both lipids show a condensation (decrease of the methylene stretching vibration wavenumbers) at the same surface pressure during compression, they must be considered miscible. If the LE/LC transition pressure differs between both lipids, they demix at least partially. These measurements can also answer the question whether DPPC and PHPC contribute differently to both transitions observed in the isotherms. Furthermore, it can be deduced whether lipid chain condensation/ordering is involved in the upper plateau, i.e., if this plateau can also be considered as phase transition plateau.

In Figure 8, the frequencies of $v_{s}\left(C_{2}\right)$ and $v_{s}\left(C_{2}\right)$ are plotted together with the compressibility of the monolayer for the mixture $x(\mathrm{PHPC})=0.75$, which exhibits positive deviation from

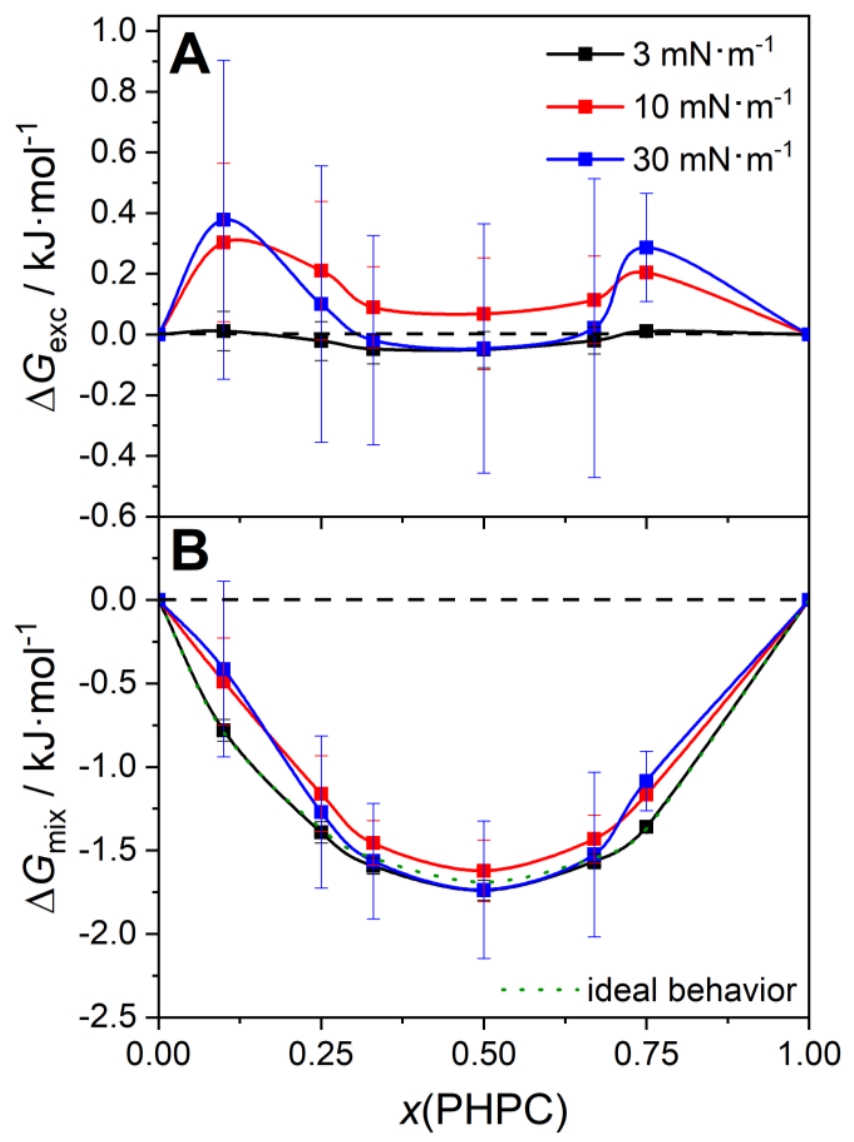

Figure 7. Thermodynamic parameters $\Delta G_{\text {exc }}\left(\right.$ A) and $\Delta G_{\text {mix }}(B)$ of DPPC/PHPC mixed monolayers as a function of mixture composition at $20{ }^{\circ} \mathrm{C}$ (a dashed line at $0 \mathrm{~kJ} \mathrm{~mol}^{-1}$ in both panels and $\Delta G_{\text {id }}$ as a green dotted line in $\mathbf{B}$ were added for clarity). ideal mixing behaviour (compare to Figure 7). The corresponding plots of all other mixing ratios are shown in Figure $\mathbf{S 1 9 .}$ Note that the phase transition surface pressure is increased in comparison to the measurements shown in Figure 5, as a result of one mixing component being chain-perdeuterated (see Figure S20). Deuteration of the lipids' acyl chains has a significant effect on the phase transition surface pressure as well as the main phase transition temperature in aqueous suspension. ${ }^{36,37}$ In all measured mixed monolayers, the lower transition appears to be shifted to higher $\pi$-values, that is, no plateau at the pure PHPC's transition pressure is detectable. This is an indication for at least partial miscibility. Like the band position of the $\mathrm{CH}_{2}$ stretching vibrations, also $v_{s}\left(C_{2}\right)$ and $v_{\text {as }}\left(C_{2}\right)$ are indicative of the trans/gauche ratio in the respective alkyl chains and can therefore be used to detect phase transitions involving chain melting. For the presented $x(\mathrm{PHPC})=0.75$ mixture (just like for all other compositions), we found that both lipids undergo a common phase transition and that at the plateau at lower $\pi$, a significant frequency shift, i.e. condensation of the lipids, occurs simultaneously. The second transition at higher surface pressure is connected to a comparatively small decrease in $\mathrm{CH}_{2} / \mathrm{CD}_{2}$ stretching vibrational frequencies. This leads to the conclusion that lipid chain condensations have only minor contribution to this second transition. When chain-deuterated DPPC is used, the two plateaus are only discernible for mixtures of $x(\mathrm{PHPC}) \geq 0.33$. In the isotherms of mixtures containing less PHPC, both transitions overlay which results in one broadened $C_{S}$ curve (compare to Figure S19).

A comparative examination at both methylene stretching vibrational bands $\left(\mathrm{CH}_{2}\right.$ as well as $\left.\mathrm{CD}_{2}\right)$ and at the phosphate stretching vibrational bands using PCA shows simultaneous transitions of the deuterated and non-deuterated lipid chains but a delayed transition in the phosphate headgroup (Figure S21 and Figure S22). After a concomitant change of the PC 1 scores in all three spectral ranges at the onset of the first plateau, the most pronounced changes in the phosphate vibrations are found at slightly elevated surface pressures, which could correlate with the surface pressure at the second plateau in the isotherms. In addition, the phosphate stretching

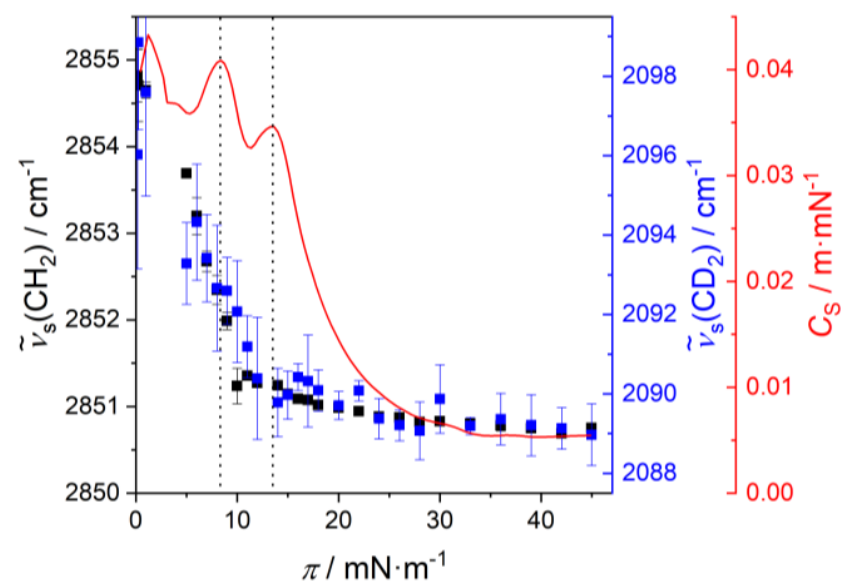

Figure 8. Comparison of $v_{s}\left(\mathrm{CH}_{2}\right)$ (black squares) and $v_{s}\left(\mathrm{CD}_{2}\right)$ (blue squares) during compression of a monolayer of an PHPC/DPPC-d62 mixture $(x(\mathrm{PHPC})=0.75)$ at $20^{\circ} \mathrm{C}$, plotted together with $C_{S}$ (red line). (To guide the eye, dotted lines are drawn at both maxima of $C_{\mathrm{s}}$, i.e. the $\pi_{\text {plateau. }}$ ) 
vibrations shows another, albeit less pronounced, change in the range between 20 and $30 \mathrm{~m} \mathrm{~N} \mathrm{~m}^{-1}$. This might be correlated with a headgroup re-orientation. In any case, it shows that transitions in the headgroup region may exist that are independent of the chain condensation. However, since we do not have enough complementary data about this transition, we refrain from further speculation about its origin.

By combining the results of all experiments performed in this study regarding miscibility of DPPC and PHPC, we conclude that there is substantial evidence that the two lipids are miscible in all mixing ratios but might show deviations from ideal miscibility. From IRRAS measurements of mixtures of PHPC and DPPC- $d_{62}$ it is found that the upper plateau does not involve a significant amount of chain ordering as it would occur in lipid LE/LC phase transitions. By employing epifluorescence microscopy, we observed two stable co-existing monolayer phases during the LE/LC phase transition (at the lower plateau). However, the full nature of the second plateau appearing only in the mixtures of both substances could not be unravelled completely.

\section{Conclusions}

In this work, we present studies of pure monolayers of the structurally related phospholipids DPPC and PHPC using $\pi-A_{\text {mol }}$ isotherms and IRRAS. In addition, we provide a detailed characterization of mixed DPPC/PHPC monolayers through their isotherms using epifluorescence microscopy, the surface phase rule, evaluation of thermodynamic mixing parameters, and IRRAS.

The isotherms of both pure lipid monolayers are comparable and differ only slightly in their phase transition pressures and LC phase compressibilities. DPPC and PHPC exhibit comparable alkyl chain trans/gauche ratios in their corresponding LE and LC phases and both form hexagonal, ordered LC phases above their LE/LC phase transition. We find that the substitution of the ester linkage at the $s n-2$ chain by an ether linkage causes several changes in lipid monolayer organization despite both lipids showing similar $\pi-A_{\text {mol }}$ isotherms. PHPC, when compared to DPPC, exhibits:

- a smaller chain tilt angle at surface pressures of $20 \mathrm{mN} \mathrm{m}^{-1}$ and above,

- stronger hydration of the carbonyl group independent of $\pi$

- stronger hydration of the phosphate group in the LE phase,

- increased headgroup ordering, and

- strong attenuation of the $\mathrm{CH}_{2}$ wagging band progressions independent of $\pi$.

These findings lead us to the conclusion that the glycerol moiety of PHPC adopts an orientation parallel to the water surface, which is different from the orientation of the glycerol of DPPC or other unsubstituted 1,2-diester phosphocholines. Similar orientational differences have been found in aqueous suspensions of the lipids before.
In the second part of this study, we present plateaus in the $\pi-A_{\mathrm{mol}}$ isotherms of mixed monolayers containing DPPC and PHPC, that do not appear in the isotherms of either of the pure lipids. The miscibility studies of mixed monolayers in the full mixing range can be interpreted in terms of non-ideal mixing behaviour but no demixing in the LE or LC phase as detected by epifluorescence microscopy and being confirmed by mixing energy calculations. Further characterization of the mixing behaviour by IRRAS shows that the upper plateau does not involve significant ordering of lipid chains. The cause for existence of the upper plateau remains unclear from our experiments but seems to involve changes in headgroup hydration. It is likely to originate from the geometrical differences (for example headgroup re-orientation) found for both pure lipid monolayers.

\section{Author Contributions}

Conceptualization M.H., C.S., and D.H.; Data curation M.H., S.D., C.S., and D.H.; Formal analysis M.H. and C.S.; Funding acquisition D.H.; Investigation M.H., S.D., and C.S.; Methodology S.D. and C.S.; Project administration C.S. and D.H.; Resources S.D., C.S., and D.H.; Software C.S.; Supervision S.D., C.S., and D.H.; Validation M.H. and C.S.; Visualization M.H., S.D., and C.S.; Writing - original draft M.H., S.D., C.S., and D.H.; Writing - review \& editing S.D., C.S., and D.H..

\section{Conflicts of interest}

There are no conflicts to declare.

\section{Acknowledgements}

We thank Kai Gruhle (MLU Halle-Wittenberg, Institute of Pharmacy) for his support during the synthesis of PHPC. M.H. thanks the Fonds der Chemischen Industrie (FCI) for their support through a Kekulé scholarship.

\section{Appendix 1}

The commonly used surface phase rule in monolayers is given by: 9,34

$$
F=\left(C_{b}+C_{m}\right)-\left(P_{b}+P_{m}\right)+3
$$

with $F$ degrees of freedom, $C_{\mathrm{b}}$ and $C_{\mathrm{m}}$ being bulk and monolayer components, respectively, and $P_{\mathrm{b}}$ and $P_{\mathrm{m}}$ being bulk and monolayer phases, respectively.

With $C_{b}+C_{m}=4$ (water, air, and two lipids) it reduces to:

$$
F=7-P
$$

with $P=P_{b}+P_{m}$. Thus, $F$ can be maximal six and is a subset of $\left\{p, T, \pi, x_{\text {monolayer }}, X_{\text {air }}, X_{\text {subphase }}\right\}$.

Assuming that bulk und monolayer components do not mix, which is given for a Langmuir monolayer, ${ }^{35}$ and that the bulk phases (air and the aqueous subphase) are always pure, it holds: 
$x_{\text {air }}=x_{\text {subphase }}=1$ (i.e., no degrees of freedom) and $C_{\mathrm{b}}=P_{\mathrm{b}}$. Thus, eq. (7) simplifies to:

$$
F=C_{m}-P_{m}+3=5-P
$$

where $F$ is a subset of $\left\{p, T, \pi, x_{\text {monolayer }}\right\}$. Assuming additionally that the bulk pressure $(p)$ does not influence the number of monolayer phases, $F$ is decreases further by one:

$$
F=C_{m}-P_{m}+2=4-P
$$

with $F$ being a subset of $\left\{T, \pi, x_{\text {monolayer }}\right\}$. Eq. (10) corresponds to the classical Gibbs phase rule applied to an insoluble monolayer (with two lipid monolayer components).

\section{References}

1. J. L. C. M. van de Vossenberg, A. J. M. Driessen and W. N. Konings, Extremophiles, 1998, 2, 163-170.

2. J.-H. Fuhrhop and T. Wang, Chemical Reviews, 2004, 104, 2901-2937.

3. M. de Rosa, A. Gambacorta and A. Gliozzi, Microbiological Reviews, 1986, 50, 70-80.

4. A. Blume, S. Drescher, G. Graf, K. Kohler and A. Meister, Adv Colloid Interface Sci, 2014, 208, 264-278.

5. R. N. A. H. Lewis, W. Pohle and R. N. McElhaney, Biophysical Journal, 1996, 70, 2736-2746.

6. G. Brezesinski, A. Dietrich, B. Struth, C. Böhm, W. G. Bouwman, K. Kjaer and H. Möhwald, Chemistry and Physics of Lipids, 1995, 76, 145-157.

7. G. Brezesinski, A. Dietrich, B. Dobner and H. Möhwald, Progress in Colloid and Polymer Science, 1995, 98, 255-262.

8. P. Mattjus, R. Bittman and J. P. Slotte, Langmuir, 1996, 12, 1284-1290.

9. H.-D. Dörfler, Advances in Colloid and Interface Science, 1990, 31, 1-110.

10. A. Blume, ChemTexts, 2018, 4, 3/1-25

11. S. W. H. Shah, C. Schwieger, J. Kressler and A. Blume, Chemistry and Physics of Lipids, 2020, 230, 104918.

12. M. Hoffmann, S. Drescher, C. Ihling, D. Hinderberger and C. Schwieger, Langmuir, 2020, 36, 12804-12815.

13. C. Schwieger, A. Meister, S. Daum, A. Blume and K. Bacia, Polymers, 2017, 9, 612/611-625.

14. S. Schrettl, C. Stefaniu, C. Schwieger, G. Pasche, E. Oveisi, Y. Fontana, A. Fontcuberta i Morral, J. Reguera, R. Petraglia, C. Corminboeuf, G. Brezesinski and $\mathrm{H}$. Frauenrath, Nat Chem, 2014, 6, 468-476.

15. C. Schwieger, B. Chen, C. Tschierske, J. Kressler and A. Blume, Journal of Physical Chemistry B, 2012, 116, 1224512256.

16. H. Abdi and L. J. Williams, Wiley Interdisciplinary Reviews: Computational Statistics, 2010, 2, 433-459.

17. R. Mendelsohn, J. W. Brauner and A. Gericke, Annual Review of Physical Chemistry, 1995, 46, 305-334.

18. A. Blume, W. Hübner and G. Messner, Biochemistry, 1988, 27, 8239-8249.

19. P. Scholtysek, Z. Li, J. Kressler and A. Blume, Langmuir, 2012, 28, 15651-15662.

$20 . \quad$ R. N. A. H. Lewis, R. N. McElhaney, W. Pohle and H. H. Mantsch, Biophysical Journal, 1994, 67, 2367-2375.

21. R. Mendelsohn, G. Mao and C. R. Flach, Biochim Biophys Acta, 2010, 1798, 788-800.
R. N. A. H. Lewis and R. N. McElhaney, Biochim Biophys Acta, 2013, 1828, 2347-2358.

of Lipids, 1998, 96, 125-139.

24.

C. R. Flach, A. Gericke and R. Mendelsohn, Journal of Physical Chemistry B, 1997, 101, 58-65.

25. L. Senak, D. Moore and R. Mendelsohn, Journal of Physical Chemistry, 1992, 96, 2749-2754.

26. T. M. Bayerl, R. K. Thomas, J. Penfold, A. Rennie and E. Sackmann, Biophysical Journal, 1990, 57, 1095-1098.

27. E. N. Serrallach, R. Dijkman, G. H. de Haas and G. G. Shipley, Journal of Molecular Biology, 1983, 170, 155-174.

28. G. Büldt and G. H. de Haas, Journal of Molecular Biology, 1982, 158, 55-71.

29. A. Weinberger, R. Tanasescu, C. Stefaniu, I. A. Fedotenko, F. Favarger, T. Ishikawa, G. Brezesinski, C. M. Marques and A. Zumbuehl, Langmuir, 2015, 31, 1879-1884.

30. I. A. Fedotenko, C. Stefaniu, G. Brezesinski and A. Zumbuehl, Langmuir, 2013, 29, 9428-9435.

31. H. Akutsu, M. Ikematsu and Y. Kyogoku, Chemistry and Physics of Lipids, 1981, 28, 149-158.

32. F. Neuhaus, D. Mueller, R. Tanasescu, C. Stefaniu, P. L. Zaffalon, S. Balog, T. Ishikawa, R. Reiter, G. Brezesinski and A. Zumbuehl, Soft Matter, 2018, 14, 3978-3986.

33. P. Krüger and M. Lösche, Physical Review E, 2000, 62, 70317043.

34. M. I. Sández Macho, A. Gil Gonzalez and A. Suárez Varela, J Colloid Interface Sci, 2001, 235, 241-246.

35. R. Smith and C. Tanford, J. Mol. Biol., 1972, 67, 75-83.

36. T. Brumm, C. Naumann, E. Sackmann, A. R. Rennie, R. K. Thomas, D. Kanellas, J. Penfold and T. M. Bayerl, European Biophysics Journal, 1994, 23, 289-295.

37. N. O. Petersen, P. A. Kroon, M. Kainosho and S. I. Chan, Chemistry and Physics of Lipids, 1975, 14, 343-349. 